\title{
Methotrexate (MTX) Plus Hydroxychloroquine versus MTX Plus Leflunomide in Patients with MTX-Resistant Active Rheumatoid Arthritis: A 2-Year Cohort Study in Real World
}

This article was published in the following Dove Press journal:

Journal of Inflammation Research

\author{
Le Zhang $\mathbb{( D}^{1,2, *}$ \\ Fangfang Chen ${ }^{2, *}$ \\ Shikai Geng $\mathbb{D}^{2, *}$ \\ Xiaodong Wang ${ }^{2}$ \\ Liyang $\mathrm{Gu}^{2}$ \\ Yitian Lang' \\ Ting $\mathrm{Li}^{2}$ \\ Shuang $\mathrm{Ye}^{2}$ \\ 'Department of Pharmacy, Ren Ji \\ Hospital, Shanghai Jiaotong University \\ School of Medicine, Shanghai, People's \\ Republic of China; ${ }^{2}$ Department of \\ Rheumatology, Ren Ji Hospital, Shanghai \\ Jiaotong University School of Medicine, \\ Shanghai, People's Republic of China \\ *These authors contributed equally to \\ this work
}

Correspondence: Shuang Ye; Ting Li Department of Rheumatology, Ren Ji Hospital, Shanghai Jiaotong University School of Medicine, Shanghai, People's Republic of China

$\mathrm{Tel}+86-2134506393$

Fax +86-2163363475

Email ye_shuang2000@163.com;

leeting007@|63.com
Purpose: To compare the efficacy, safety, and cost-effectiveness of methotrexate (MTX) plus hydroxychloroquine (HCQ) vs MTX plus leflunomide (LEF) in established rheumatoid arthritis (RA) with inadequate response to MTX monotherapy in a real-world Chinese cohort.

Patients and Methods: A prospective RA cohort ( $\mathrm{n}=549)$ was screened with eligible patients who had inadequate response (disease activity score in 28 joints using erythrocyte sedimentation rate, DAS28-ESR>3.2) to initial MTX monotherapy and subsequently received either MTX + HCQ or MTX+LEF. Propensity score matching (PSM) was applied to adjust the possible baseline confounders between two groups. The primary outcome was the proportion of patients achieving first remission (DAS28-ESR<2.6) during follow-up by log rank test. Secondary outcomes were changes of DAS28, glucocorticoids (GCs) exposure, safety, cost-effectiveness, sustained remission, and low disease activity (LDA) rate after 24-month follow-up.

Results: Overall, 222 eligible patients were subjected to the aforementioned two treatment protocols (MTX+HCQ, n=102; MTX+LEF, $n=120$ ). After PSM adjustment, 97 patients in each group were analyzed. A higher remission rate was observed in the MTX+HCQ group than in the MTX+LEF group $(70.1 \%$ vs $56.7 \%, P=0.048)$. The median time to remission was 11 and 16 months in the two groups, respectively. At the endpoint, more patients achieved remission (46.8\% vs $32.5 \%, P=0.063$ ) and maintained sustained LDA in the HCQ group $(53.2 \%$ vs $38.6 \%, P=0.062)$ and also more patients withdrew GCs in this group (32\% vs $16.7 \%, P=0.053$ ) than those in the LEF group. Safety profiles were non-alarming, with no significant difference between the two groups. The incremental cost-effectiveness ratio yielded by MTX+HCQ over MTX+LEF was $\$ 1,111.8$ per quality-adjusted life-year (QALY), within the cost-effective threshold set as the per capita gross domestic product (GDP) of China.

Conclusion: The MTX+HCQ combination was seemingly superior to MTX+LEF in a realworld cohort of Chinese RA patients with inadequate response to methotrexate monotherapy in respect of the efficacy and cost-effectiveness.

Keywords: hydroxychloroquine, leflunomide, methotrexate-resistant, rheumatoid arthritis, efficacy, cost-effectiveness

\section{Introduction}

Methotrexate (MTX) is recommended as the cornerstone of initial treatment for rheumatoid arthritis (RA). ${ }^{1}$ However, approximately $50 \%$ patients who experience 
failure in the first course of methotrexate monotherapy ${ }^{2-4}$ will require an escalated treatment according to the treat-totarget strategy. ${ }^{5,6}$ Previous clinical trials have compared the effectiveness of different approaches among such MTX inadequate responders, which mainly can be divided into two protocols, ie, combination of conventional synthetic disease-modifying antirheumatic drugs (csDMARDs) with or without the use of glucocorticoids, ${ }^{7-9}$ or escaping to more powerful, yet costly, targeted therapies. ${ }^{10-13}$

Previous studies demonstrated that the global disease burden of RA ranked fifth in musculoskeletal diseases, and its average annual direct cost in China could reach $\$ 3,069 .{ }^{14,15}$ The increasing disease burden in light of pharmacoeconomic analysis is at least partially relevant to the emerging targeted therapies. Therefore, in the real-world practice, to give the csDMARDs a "second chance" is always a serious consideration for both health providers and patients.

In this regard, the inevitable next question is, "what would be the best combination of csDMARDs?" The "triple therapy" combining MTX, sulfasalazine (SSZ), and hydroxychloroquine (HCQ) is the most representative protocol with abundant evidence. ${ }^{4,16,17}$ Other combinations, such as MTX + SSZ and MTX + HCQ have also been extensively studied. ${ }^{18-20}$ Leflunomide (LEF), with a comparable potency to MTX alone, has become an alternative option. $^{21,22}$ To answer the question in a real-world setting, the compliance issue should always be taken into account because non-compliance will largely impede the treat-totarget achievement. ${ }^{23}$ According to previous data, ${ }^{24}$ the rule of thumb is "the more pills, the less compliance".

Therefore, we investigated two commonly used simple combinations in real-world, MTX+HCQ vs MTX+LEF, in a prospective cohort of established RA with inadequate response to initial MTX monotherapy. The aim of this study is to fill the gap that the efficacy, safety, and costeffectiveness of MTX+HCQ and MTX+LEF has never been compared, and moreover it is highly probable that a randomized head-to-head comparison of these two combinations will never be performed.

\section{Materials and Methods Study Design}

This observational cohort was established following the OPCSP trial (ClinicalTrials.gov ID: NCT03024307), a randomized trial on a compliance improvement program among rheumatic patients by a multidisciplinary team. The research protocol was approved by Shanghai Jiaotong
University School of Medicine, Renji Hospital Ethics Committee (approval No.[2016]216K). All participating patients provided written informed consent.

Eligible patients' data were extracted when the following inclusion criteria were met; 1) all patients fulfilled 2010 criteria for RA of the American College of Rheumatology or European League Against Rheumatism; ${ }^{25}$ 2) they had an inadequate response to initial MTX monotherapy, defined as DAS28-ESR>3.2 (28-joint disease activity score using erythrocyte sedimentation rate) after receiving MTX for at least 6 months with a stable route of administration and more than $10 \mathrm{mg}$ weekly for at least 3 months prior to the baseline visit; and 3) subsequently using either MTX+HCQ or MTX+LEF treatments according to routine clinical practice. Patients were excluded if they were illiterate, had severe mental disorders, had serious physical constraints, or were over the age of 85 . Nonsteroidal anti-inflammatory agents (NSAIDs) and glucocorticoids (GCs) were allowed to administrate at stable doses if needed. If an improvement in disease activity was achieved by the judgment of the investigator, the dose of GCs could be reduced, but tapering could not be changed within the 2 weeks before assessment. A single intra-articular injection of glucocorticoids was allowed during the study; the injected joint was considered to be non-evaluable for subsequent study visits.

\section{Outcome Assessment}

DAS28 scores, laboratory values, quality-of-life assessments by EQ5D (EuroQol five-dimensions questionnaire) ${ }^{26}$ and medications (eg, NSAIDs and GCs) during the follow-up were recorded. The primary outcome was the proportion of patients having reached at least one remission (DAS28ESR $<2.6$ ) over 2 years among propensity score matched (PSM) patients, ${ }^{20,27}$ the low disease activity (LDA) rate and treatment retention were also calculated. Switching therapies were documented for up to 2 years. When switching of therapies occurred, the original therapy was defined as failure. Secondary outcomes were the changes of DAS28, average daily exposure to GCs, proportion of patients who withdrew from GCs, and cost-effectiveness by the available extended 2-year data in matched patients. Data from patients who completed 2-year follow-up were analyzed to determine whether the remission and LDA response observed over 12 months of two groups would be sustained over 24 months. The participants reported the possible side-effects of the treatment at each follow-up and all records were included in the safety analysis whether the participants completed the 2-year follow-up schedules or not. 


\section{Statistical Analysis}

The comparisons between the study groups were performed with the use of Student's $t$-test for continuous measures and the chi-square test (or Fisher's exact test) for categorical measures. The primary comparison between two groups was the time until first moment of DAS28 remission by Kaplan-Meier survival analysis with Log rank testing. The rate of LDA and retention of the treatment were also compared by Kaplan-Meier survival analysis with Log rank testing. The changes of DAS28 over time were compared by specifying a linear mixed model with treatment and time as fixed factors, and was performed with baseline DAS28 score as a covariate. As the visit schedules were slightly different between both cohorts, only baseline, 12-month, and 24-month data were included for the analysis of the secondary outcomes and we allowed a 2-month window (the closest information to the time within 2 months before or after this time). Results were presented as all observed data, without imputation for missing values. All statistical calculations were performed using the statistical software package IBM SPSS version 25.0 for mac (IBM, Armonk, NY, USA). For all tests, $P<0.05$ was considered to be significant.

Considering the baseline difference caused by possible selection bias, PSM was applied to adjust the baseline confounders. The matching was based on prognostic factors, including number of previous DMARDs used, daily GCs exposure dose, and DAS28-ESR. ${ }^{28,29}$ The nearest neighbor method was used for 1:1 matching, and the caliper value was set to 0.02 .

\section{Cost-Effectiveness Analysis}

We established a decision tree model to evaluate the mean between-group difference in costs and quality-adjusted lifeyears (QALYs) gained over 24 months among the patients completing 2-year follow-up without switching therapy. Since two brands of hydroxychloroquine were included in this study, we calculated the weighed price of a hydroxychloroquine treatment group according to the proportion of two bands in the study. We estimated QALYs from the health utility value, derived from EQ-5D questionnaire responses and assessed value of the EQ-5D using Chinese time trade-off values. ${ }^{26,30}$ An incremental cost-effectiveness ratio was calculated by dividing the cost difference by the QALY difference per pair of treatment schemes. ${ }^{15}$ The 10,000 iterations Monte Carlo simulation was implemented to get the cost-effectiveness acceptability curve to analyze the preferred treatment group between different willingness-to-pay (WTP) thresholds. The WTP threshold under the per capita gross domestic product (GDP) of China was considered cost-effective, \$10,279 in 2019.

\section{Results \\ Cohort Baseline}

Two hundred and twenty-two out of 549 patients of the cohort were eligible participants, and 102 of them were administered MTX+HCQ, and $120 \mathrm{MTX}+\mathrm{LEF}$ (Figure 1). Baseline demographic and clinical characteristics of participants are summarized in Table 1. In this study, two care programs were proportionately distributed in two analyzed groups. The average disease duration was 3 years and all of them had a diagnosis of RA longer than 1 year. The proportion of patients with positive ACPA and/or RF was over $85 \%$. The disease activity was similar in both groups, such as DAS28-ESR (4.3 vs 4.4, $P=0.394$ ). The proportion of patients with moderate disease activity $(80.4 \%$ vs $80.8 \%, P=0.934)$ and high disease activity $(19.6 \%$ vs $19.2 \%, P=0.934)$ were similar between the two groups. The average weekly dose of MTX was $10 \mathrm{mg}$, which was equally distributed in the two groups. The average daily dose of HCQ and LEF were $200 \mathrm{mg}$ and $10 \mathrm{mg}$, respectively. After PSM, the distribution of baseline parameters was further balanced. Sixty-two patients $(63.9 \%)$ received GCs in the HCQ group at an average daily dose of $9 \mathrm{mg}$, whereas 75 patients $(77.3 \%)$ received GCs in the LEF group at an average daily dose of $8.5 \mathrm{mg}(P=0.464)$.

\section{Efficacy}

Adjusted by PSM, 97 participants were matched in both groups and the proportion at some point during follow-up achieving DAS28 remission were higher in the MTX + HCQ group ( $70.1 \%$ vs $56.7 \%, P=0.048$ ) (Figure 2A), while the LDA rate was not significantly different (76.3\% vs $69.1 \%, P=0.125$ ) (Figure $2 \mathrm{~B}$ ). Median time to remission was 11 months in the MTX+HCQ group (95\% confidence interval $[\mathrm{CI}]=5.7-16.3)$ vs 16 months in the MTX+LEF group (95\% CI $=6.8-25.2)$; the median time to LDA was 6 months in the HCQ group $(95 \%$ $\mathrm{CI}=4.8-7.2)$ and 10 months in the LEF group (95\% $\mathrm{CI}=5.7-14.3$ ), respectively. The drug retention rate of two regimens was similar $(88.7 \%$ vs $82.5 \%, P=0.292)$ (Figure 2C).

The secondary efficacy endpoints at 24 months are illustrated in Table 2. Among the 162 matched patients who 


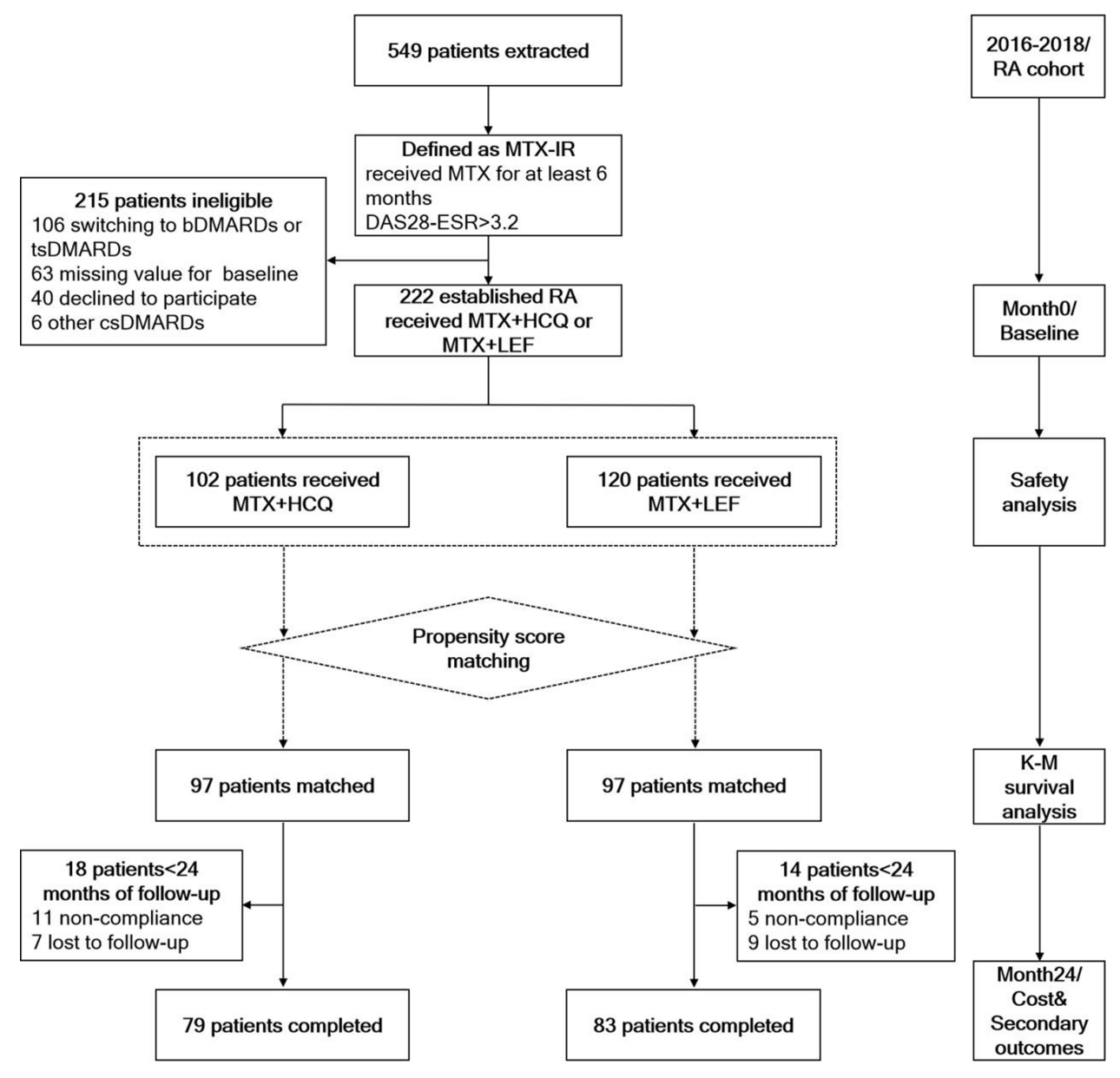

Figure I Flow diagram of the analyzed study population.

Abbreviations: RA, rheumatoid arthritis; DAS28-ESR, disease activity score in 28 joints using erythrocyte sedimentation rate; MTX, methotrexate; MTX-IR, methotrexate inadequate response; DMARDs, disease-modifying antirheumatic drugs; bDMARDs, biological DMARDs; tsDMARDs, targeted synthetic DMARDs; csDMARDs, conventional synthetic DMARDs; HCQ, hydroxychloroquine; LEF, leflunomide; K-M survival analysis, Kaplan-Meier survival analysis.

completed the follow-up, the remission rate was marginally higher in the HCQ group than in the LEF group (46.8\% vs $32.5 \%, P=0.063$ ), with $25.3 \%$ of patients achieving sustained remission from 12 months to the endpoint in the HCQ group and $15.7 \%$ in the LEF group $(P=0.127)$. The LDA rate was $65.8 \%$ in the HCQ group and $56.6 \%$ in the LEF group ( $P=0.230$ ), with $53.2 \%$ of patients achieving sustained LDA in the HCQ group and $38.6 \%$ in the LEF group $(P=0.062)$. The mean score of DAS28-ESR was 2.7 in the MTX+HCQ group, whereas it was 2.9 in the MTX + LEF group $(P=0.246)$. The net changes of DAS28-ESR score over 2 years were similar with a slight, yet significant, greater DAS28-ESR reduction in the MTX+HCQ group than in the MTX+LEF group $(95 \% \mathrm{CI}=0.006-0.478$, $P=0.044$ ) (Supplementary Figure S1). The VAS pain (2.6 vs $2.3, P=0.328)$ and EQ5D-index $(0.812$ vs 0.771 , $P=0.169)$ were similar between groups. In respect of the concomitant medication, a smaller proportion of patients were prescribed with GCs at 24 months in the HCQ group (45.6\% vs $69.9 \%, P=0.002)$. In addition, more patients tapered off and finally withdrew GCs in the MTX+HCQ group $(16,32.0 \%)$ than in the MTX+LEF group (11, $16.7 \%)$ at the endpoint $(P=0.053)$, while no significant difference was observed about the daily dose of GCs among the 94 patients $(58.0 \%)$ still receiving GCs $(6.7 \mathrm{mg}$ vs $6.2 \mathrm{mg}, P=0.592)$.

\section{Cost-Effectiveness Analysis}

Medication costs and QALYs for each treatment group are presented in Table 3. The medication cost for 2 years in the MTX+HCQ group was $\$ 547.2$ and \$516.4 in the MTX + LEF group. The patients gained an average 1.603 QALYs in MTX+HCQ group and 1.575 in the MTX+LEF group. The incremental cost-effectiveness ratio (ICER) yielded by MTX+HCQ therapy was $\$ 1,111.8$ per QALY, compared with the MTX+LEF therapy. Cost-effectiveness 
Table I Baseline Characteristics of Patients Before and After Propensity Score Matching

\begin{tabular}{|c|c|c|c|c|c|c|}
\hline \multirow[t]{2}{*}{ Characteristics } & \multicolumn{3}{|c|}{ Overall Sample $(n=222)$} & \multicolumn{3}{|c|}{ Matched Patients $(n=194)$} \\
\hline & $\begin{array}{l}\text { MTX+HCQ } \\
(n=102)\end{array}$ & $\begin{array}{l}\text { MTX+LEF } \\
(n=\mid 20)\end{array}$ & $P$-value & $\begin{array}{l}\text { MTX+HCQ } \\
(n=97)\end{array}$ & $\begin{array}{l}\text { MTX+LEF } \\
(n=97)\end{array}$ & $P$-value \\
\hline Age, mean (SD), years & $51.0(14.2)$ & $53.3(10.9)$ & 0.179 & $50.2(13.8)$ & $53.3(10.8)$ & 0.083 \\
\hline Female, n (\%) & $85(83.3)$ & $88(73.3)$ & 0.077 & $81(83.5)$ & $72(74.2)$ & 0.113 \\
\hline BMI, mean (SD) & $21.5(3.0)$ & $22.2(3.0)$ & 0.090 & $21.6(3.0)$ & $22.0(3.0)$ & 0.282 \\
\hline Smoking history, n (\%) & $7(6.9)$ & $7(5.8)$ & 0.788 & $7(7.2)$ & $5(5.2)$ & 0.767 \\
\hline Drinking history, n (\%) & $6(5.9)$ & $4(3.3)$ & 0.519 & $6(6.2)$ & $\mathrm{I}(\mathrm{I} .0)$ & 0.118 \\
\hline Disease duration, median (IQR), years & $3(2-6)$ & $3(2-8)$ & 0.556 & $5.5(6.1)$ & $5.3(5.1)$ & 0.780 \\
\hline Comorbidities, n (\%) & $45(44.1)$ & $45(37.5)$ & 0.526 & $42(43.3)$ & $31(32.0)$ & 0.103 \\
\hline 0 & $57(55.9)$ & $75(62.5)$ & & $54(55.7)$ & $66(68.0)$ & \\
\hline 1 & $32(31.4)$ & $28(23.3)$ & & $31(32.0)$ & $17(17.5)$ & \\
\hline 2 & $9(8.8)$ & $9(7.5)$ & & $12(12.4)$ & $14(14.4)$ & \\
\hline$\geq 3$ & $4(3.9)$ & $8(6.6)$ & & 0 & 0 & \\
\hline ACPA+*, n (\%) & 79 (85.9) & 14 (86.9) & 0.830 & $76(87.4)$ & $75(86.2)$ & 0.823 \\
\hline RF+**, n (\%) & 71 (85.5) & $81(91.0)$ & 0.264 & $70(87.5)$ & $63(90.0)$ & 0.630 \\
\hline DAS28-ESR, mean (S.D.) & $4.3(0.8)$ & $4.4(0.8)$ & 0.394 & $4.3(0.8)$ & $4.5(0.9)$ & 0.143 \\
\hline DAS28-CRP, mean (S.D.) & $3.9(0.8)$ & $3.9(0.8)$ & 0.519 & $3.8(0.7)$ & $4.0(0.8)$ & 0.189 \\
\hline MDA rate, $\mathrm{n}(\%)$ & $82(80.4)$ & $97(80.8)$ & 0.934 & $78(80.4)$ & $76(78.4)$ & 0.723 \\
\hline HDA rate, n (\%) & $20(19.6)$ & $23(19.2)$ & 0.934 & $19(19.6)$ & $21(21.6)$ & 0.723 \\
\hline EQ-5D index, mean (SD) & $0.7(0.2)$ & $0.7(0.2)$ & 0.960 & $0.7(0.2)$ & $0.7(0.2)$ & 0.789 \\
\hline GCs usage, $n(\%)$ & $64(62.7)$ & $92(76.7)$ & 0.024 & $62(63.9)$ & $75(77.3)$ & 0.058 \\
\hline GCs dosage", mean (SD), mg & $9.2(5.4)$ & $8.1(3.8)$ & 0.135 & $9.0(5.4)$ & $8.5(3.9)$ & 0.464 \\
\hline Weekly dose of MTX, mean (SD), mg & $10.6(1.9)$ & $10.4(1.6)$ & 0.450 & $10.6(1.9)$ & $10.4(1.4)$ & 0.329 \\
\hline Prior DMARDs, n (\%) & I.3 (0.6) & $1.2(0.5)$ & 0.318 & $1.2(0.5)$ & I.2(0.5) & 0.888 \\
\hline bDMARD, n (\%) & $2(2.0)$ & 0 & 0.210 & $2(2.1)$ & 0 & 0.497 \\
\hline Use of NSAID, n (\%) & $7(6.9)$ & $7(5.8)$ & 0.788 & $7(7.2)$ & $7(7.2)$ & 1.000 \\
\hline Multidisciplinary care, n (\%) & $60(58.8)$ & $68(56.7)$ & 0.746 & $58(59.8)$ & $57(58.8)$ & 0.884 \\
\hline
\end{tabular}

Notes: $* 23$ Missing data were excluded in calculating the positive proportion. ${ }^{*} * 50$ Missing data were excluded in calculating the positive proportion. ${ }^{\#}$ Average daily dose of GCs was calculated in prescribed patients.

Abbreviations: IQR, interquartile range; MTX, methotrexate; HCQ, hydroxychloroquine; LEF, leflunomide; BMI, body mass index; ACPA, anticitrullinated protein antibody; RF, rheumatoid factor; DAS28-ESR, disease activity score in 28 joints using erythrocyte sedimentation rate; DAS28-CRP, disease activity score in 28 joints using c-reactive protein; MDA, moderate disease activity; HDA, high disease activity; EQ-5D, EuroQol five-dimensions questionnaire; GC, glucocorticoid; DMARDs, disease-modifying antirheumatic drugs; bDMARDs, biological DMARDs; NSAID, non-steroidal anti-inflammatory drug.

acceptability curves also indicated that MTX+HCQ was the more cost-effective when the per capita GDP of China $(\$ 10,279)$ was set as a possible willingness-to-pay threshold (Supplementary Figure S2).

\section{Safety Analysis}

There were no serious adverse effects observed during the follow-up, and also no difference in the proportion of participants who experienced comorbidity $(46.1 \%$ vs $45.8 \%, P=0.971)$ or side-effects $(22.5 \%$ vs $20.8 \%$, $P=0.870$ ) across treatment groups (Table 4). In our study cohort, the most common comorbidity with RA was osteoporosis $(19.8 \%)$, followed by cardiovascular diseases $(10.8 \%)$, digestive system diseases $(9.5 \%)$, and other rheumatic diseases (5.0\%). The most frequently reported side-effects were gastrointestinal reactions $(5.9 \%)$, followed by infections $(4.5 \%)$, hepatotoxicity $(3.6 \%)$, and leukopenia $(2.3 \%)$.

\section{Discussion}

In this observational cohort study, the combination of MTX plus HCQ or LEF was compared in established RA who had inadequate response to MTX monotherapy with moderate or high disease activity. After adjustment by PSM, the first time to remission over 2 years favored MTX+HCQ over MTX+LEF combination. At 24 months, we also observed a high remission rate and sustained LDA rate in the HCQ group, though the statistical difference 
A Time until remission
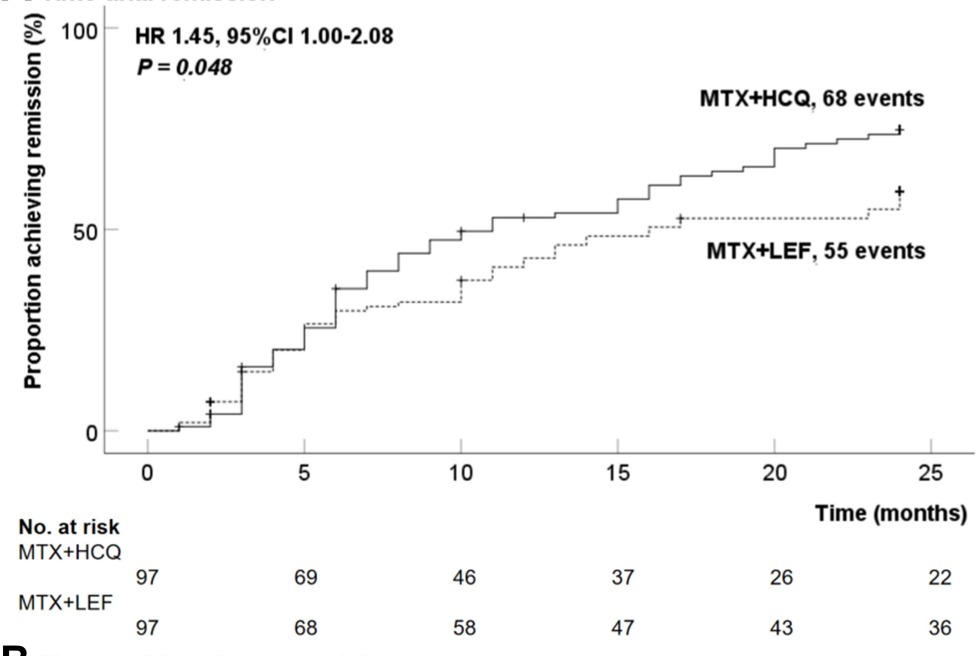

B Time until low disease activity

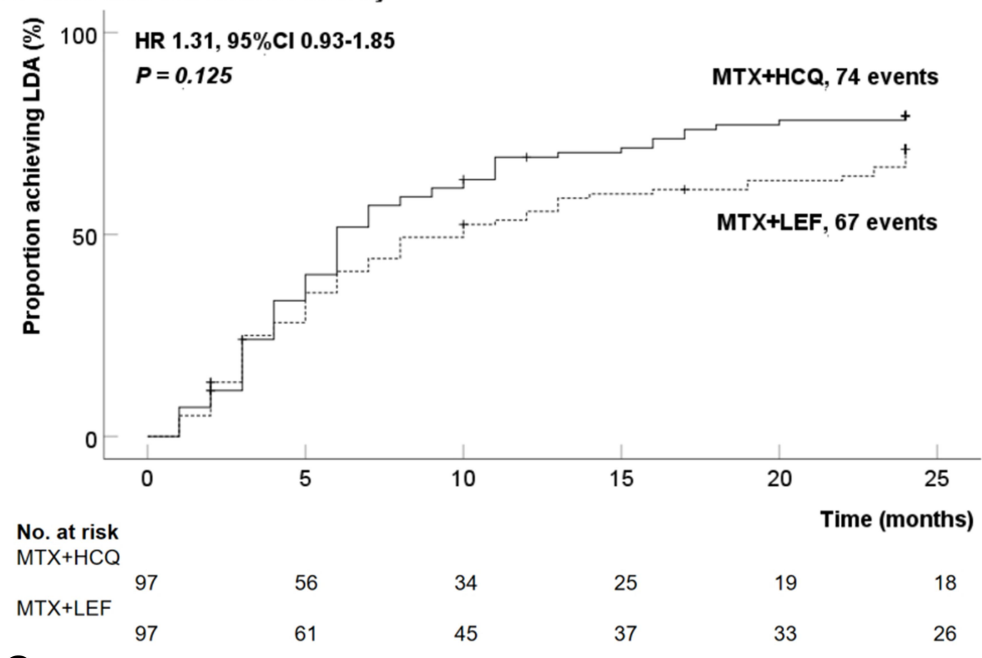

C Drug retention

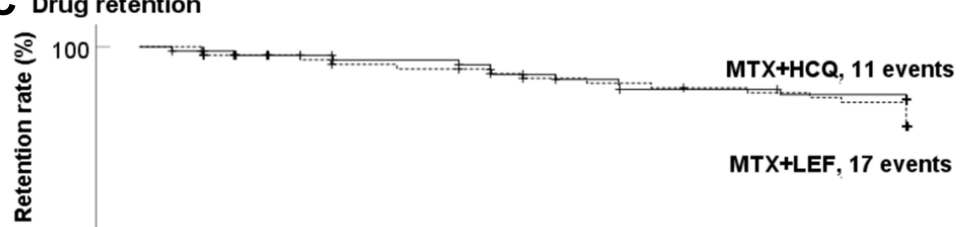

50

HR 0.67, 95\% $\mathrm{Cl} 0.32-1.41$

$P=0.292$

0

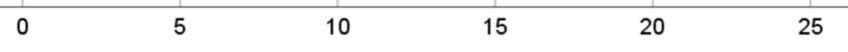

No. at risk

$\mathrm{MTX}+\mathrm{HCQ}$

$\begin{array}{rrrrrr}\text { MTX+LEF } & 97 & 82 & 72 & 69 & 68 \\ 97 & 86 & 83 & 77 & 74 & 67\end{array}$

Figure 2 Kaplan-Meier curve for time to first remission (A), LDA (B), and drug retention (C) among matched patients. The solid line represents MTX+HCQ, and the dashed line represents MTX+LEF.

Abbreviations: MTX, methotrexate; HCQ, hydroxychloroquine; LEF, leflunomide; LDA, low disease activity; HR, hazard ratio; Cl, confidence interval. 
Table 2 Secondary Efficacy Endpoints

\begin{tabular}{|c|c|c|c|c|c|c|c|c|c|}
\hline \multirow[t]{2}{*}{ Measure } & \multicolumn{3}{|l|}{ Month 0} & \multicolumn{3}{|l|}{ Month 12} & \multicolumn{3}{|l|}{ Month 24} \\
\hline & $\begin{array}{l}\text { MTX } \\
+H C Q \\
(n=97)\end{array}$ & $\begin{array}{l}\text { MTX } \\
\text { +LEF } \\
(n=97)\end{array}$ & $P$-value & $\begin{array}{l}\text { MTX } \\
+H C Q \\
(n=86)\end{array}$ & $\begin{array}{l}\text { MTX } \\
+ \text { LEF } \\
(n=87)\end{array}$ & $P$-value & $\begin{array}{l}\text { MTX } \\
+H C Q \\
(n=79)\end{array}$ & $\begin{array}{l}\text { MTX } \\
+ \text { LEF } \\
(n=83)\end{array}$ & $P$-value \\
\hline DAS28-ESR, mean (SD) & $4.3(0.8)$ & $4.5(0.9)$ & 0.143 & $2.9(1.1)$ & $3.2(1.0)$ & 0.066 & $2.7(1.1)$ & $2.9(1.1)$ & 0.246 \\
\hline DAS28-CRP, mean (SD) & $3.8(0.7)$ & $4.0(0.8)$ & 0.189 & $2.2(0.9)$ & $2.4(0.9)$ & 0.157 & $2.5(0.9)$ & $2.6(0.9)$ & 0.525 \\
\hline VAS pain, mean (SD) & $4.9(1.6)$ & $4.7(1.9)$ & 0.312 & $2.8(1.8)$ & $2.8(1.9)$ & 0.960 & $2.6(1.7)$ & $2.3(1.9)$ & 0.328 \\
\hline Remission rate, n (\%) & 0 & 0 & I & $35(40.7)$ & $30(34.5)$ & 0.399 & $37(46.8)$ & $27(32.5)$ & 0.063 \\
\hline Sustained remission rate, $\mathrm{n}(\%)$ & I & I & I & 1 & 1 & I & $20(25.3)$ & $13(15.7)$ & 0.127 \\
\hline LDA rate, n (\%) & 0 & 0 & l & $51(59.3)$ & $42(48.3)$ & 0.146 & $52(65.8)$ & $47(56.6)$ & 0.230 \\
\hline Sustained LDA rate, $\mathrm{n}(\%)$ & I & l & I & 1 & 1 & I & $42(53.2)$ & $32(38.6)$ & 0.062 \\
\hline EQ5D, mean (SD) & $0.712(0.2)$ & $0.705(0.2)$ & 0.789 & $0.796(0.1)$ & $0.765(0.2)$ & 0.137 & $0.812(0.2)$ & $0.77 \mid(0.2)$ & 0.169 \\
\hline GCs usage, $n(\%)$ & $62(63.9)$ & $75(77.3)$ & 0.058 & $46(53.5)$ & $61(70.1)$ & 0.029 & $36(45.6)$ & $58(69.9)$ & 0.002 \\
\hline Withdrawal from GCs*, n (\%) & l & l & 1 & $10(18.5)$ & $10(14.9)$ & 0.597 & $16(32.0)$ & II (I6.7) & 0.053 \\
\hline GCs dose ${ }^{\#}$, mean (SD), mg & $9.0(5.4)$ & $8.5(3.9)$ & 0.464 & $7.5(3.6)$ & $7.2(4.0)$ & 0.688 & $6.7(4.2)$ & $6.2(3.8)$ & 0.592 \\
\hline Use of NSAID, $n(\%)$ & $7(7.2)$ & $7(7.2)$ & 1.000 & $2(2.3)$ & $3(3.4)$ & 1.000 & $3(3.8)$ & $\mathrm{I}(\mathrm{I} .2)$ & 0.358 \\
\hline
\end{tabular}

Notes: *Proportion of patients who withdrew from GCs was calculated in prescribed patients still under follow-up. At 12 months, 54 patients were prescribed GC at baseline in the HCQ group and 67 in the LEF group. At 24 months, 50 patients were prescribed GC at baseline in the HCQ group and 66 in the LEF group. ${ }^{\#}$ Average daily dose of GCs was calculated in prescribed patients.

Abbreviations: MTX, methotrexate; HCQ, hydroxychloroquine; LEF, leflunomide; DAS28-ESR, disease activity score in 28 joints using erythrocyte sedimentation rate; DAS28-CRP, disease activity score in 28 joints using c-reactive protein; VAS pain, visual analogscale for pain (0-10); LDA, low disease activity; EQ-5D, EuroQol fivedimensions questionnaire; GC, glucocorticoid; NSAID, non-steroidal anti-inflammatory drug.

was at the critical value. In addition, significantly fewer patients were administered GCs in the HCQ group than that in the LEF group at the endpoint.

Previous studies in patients with inadequate response to MTX showed that the initial combination therapy of target agents and MTX appeared to be more effective than it alone, ${ }^{8,31-34}$ the triple combination of MTX+SSZ+HCQ was not inferior to etanercept plus MTX. In spite of this knowledge, considerable patients still prefer the conventional combination therapy after failure in the first course of MTX monotherapy for the sake of the cost of biological agents. ${ }^{20}$ Also, patients receiving target agents were likely to discontinue treatment for economic factors. ${ }^{23,35}$ On account of the cost and compliance issues, a combination of conventional

Table 3 Medication Costs and QALYs Over 24 Months

\begin{tabular}{|l|l|l|}
\hline Measure & $\begin{array}{l}\text { MTX+HCQ } \\
(\mathbf{n = 6 8 )}\end{array}$ & $\begin{array}{l}\text { MTX+LEF } \\
\text { (n=67) }\end{array}$ \\
\hline Medication costs (US\$) & 547.2 & 516.4 \\
$\Delta$ Costs (US\$) & 30.8 & Reference \\
QALYs & 1.603 & 1.575 \\
$\triangle$ QALYs & 0.028 & Reference \\
Average C/E (\$/QALY) & 327.8 & 327.8 \\
ICER (\$/QALY) & $1,111.8$ & Reference \\
\hline
\end{tabular}

Abbreviations: MTX, methotrexate; HCQ, hydroxychloroquine; LEF, leflunomide; ICER, incremental cost-effectiveness ratio; QALY, quality-adjusted life years; Average $C / E$, average cost-effectiveness ratio.
DMARDs should be considered in the real-world setting when initial MTX monotherapy was suboptimal. However, the limited research on hydroxychloroquine mainly focused on triple combined therapy with a relatively high dose of MTX and SSZ. ${ }^{7,36}$ It was not quite feasible for real-world practice, for concerns about patient compliance and unfavorable side-effects due to the differences in ethnic adaptability, ${ }^{36}$ a maximum dose of $16 \mathrm{mg}$ MTX weekly in a Japanese population. ${ }^{1,37}$ According to our data, a combination of $10 \mathrm{mg}$ MTX weekly plus hydroxychloroquine $200 \mathrm{mg}$ daily in patients with a low body mass index (BMI) of 21.9 on average was safe and more effective than plus $10 \mathrm{mg}$ LEF daily. Indeed, a previous pharmacokinetic study had underscored a synergistic effect of the MTX+HCQ combination. ${ }^{38}$ On the other hand, the classic triple therapy of MTX $+\mathrm{SSZ}+\mathrm{HCQ}$, which bore abundant evidence in terms of efficacy; ${ }^{8,17}$ however, its compliance was problematic in Chinese patients according to our previous real-world data. ${ }^{14}$ The main reason seemed to be that patients were reluctant to take an extra 4-8 SSZ tablets each day (with a therapeutic dose of 1-2 gram/day). MTX+LEF apparently provided an alternative option; and within the dose range the safety profile was good. However, our data supported that MTX $+\mathrm{HCQ}$ had a better remission response over MTX+LEF combination at a low dose in the patients with a low BMI.

Admittedly, the average daily exposure to GCs in this study was higher than previously reported, but still within 
Table 4 Safety Outcomes $(n=222)$

\begin{tabular}{|c|c|c|c|}
\hline Measure & $\begin{array}{l}\text { MTX+HCQ } \\
(n=102)\end{array}$ & $\begin{array}{l}\text { MTX+LEF } \\
(n=\mid 20)\end{array}$ & $P$-value \\
\hline Comorbidities, n (\%) & $47(46.1)$ & $55(45.8)$ & 0.971 \\
\hline 0 & $55(53.9)$ & $65(54.2)$ & \\
\hline 1 & $33(32.4)$ & $38(31.7)$ & \\
\hline 2 & $10(9.8)$ & $9(7.5)$ & \\
\hline$\geq 3$ & $4(4.8)$ & $8(6.6)$ & \\
\hline Osteoporosis & $19(18.6)$ & $25(20.8)$ & \\
\hline Cardiovascular diseases & II (10.8) & $13(10.8)$ & \\
\hline Digestive system diseases & $10(9.8)$ & II (19.2) & \\
\hline Other rheumatic diseases & $7(6.9)$ & $4(3.3)$ & \\
\hline Endocrine system & $6(5.9)$ & $9(7.5)$ & \\
\hline Respiratory diseases & $5(4.9)$ & $6(5)$ & \\
\hline Musculoskeletal diseases & $3(2.9)$ & $3(2.5)$ & \\
\hline Hematological system diseases & $2(2.0)$ & $2(1.7)$ & \\
\hline Renal disease & $2(2.0)$ & $5(4.2)$ & \\
\hline Nervous system & 0 & $3(2.5)$ & \\
\hline Adverse effects, $n$ (\%) & $23(22.5)$ & $25(20.8)$ & 0.870 \\
\hline 0 & $79(77.5)$ & $95(79.2)$ & \\
\hline I & $20(19.6)$ & $24(20)$ & \\
\hline 2 & $3(2.9)$ & $\mathrm{I}(0.8)$ & \\
\hline Gastrointestinal response & $6(5.9)$ & $7(5.8)$ & \\
\hline Infection & $6(5.9)$ & $4(3.3)$ & \\
\hline Hepatotoxicity & $2(2.0)$ & $6(5.0)$ & \\
\hline Leukopenia & $2(2.0)$ & $3(2.5)$ & \\
\hline Musculoskeletal diseases & $3(2.9)$ & I $(0.8)$ & \\
\hline Blurred vision & $2(2.0)$ & 0 & \\
\hline Alopecia & $I(1.0)$ & $2(1.8)$ & \\
\hline Rash & 0 & $\mathrm{I}(0.8)$ & \\
\hline
\end{tabular}

Abbreviations: MTX, methotrexate; HCQ, hydroxychloroquine; LEF, leflunomide.

the recommended dose of $7.5 \mathrm{mg}$ or less. ${ }^{1}$ The tapering scheme of GCs could be optimized. GCs should primarily be used as a bridging therapy until DMARDs exhibit their efficacy and should be tapering off as rapidly as clinically feasible. However, the participants of this study preferred conservative treatment, they neither accepted target agents, nor step-up DMARDs dosage. In real-world practice, the compliance, preference, and economy of patients should always be considered when we provide therapies. Because of the inability to switch to more effective DMARD treatment, GC was administrated to control the disease activity. From our results, fewer patients were administered GCs in the HCQ group and more of them tapered off and finally withdrew it than in the LEF group at the endpoint. This result suggested that MTX+HCQ might be of more benefit than LEF for these difficult patients.

Previous cost-effectiveness analyses on RA were mainly centered on bioDMARDs vs csDMARDs. ${ }^{39-42}$ The comparison among the conventional combinations were limited, ${ }^{43}$ especially the cost-effectiveness of MTX+HCQ over MTX+LEF. This study provided direct evidence that in China mainland, the ICER yielded by MTX+HCQ was $\$ 1,111.8$ per QALY greater than MTX+LEF. Within the costeffective threshold set as the per capita GDP of China, our data indicated a favorable cost-effectiveness with MTX +HCQ combination. This analysis was of greater reference value for patients in other countries such as in South America and Europe, because the price of leflunomide in these countries was often much higher than that of hydroxychloroquine. In view of these conditions, the findings of our study on the effectiveness and economy of methotrexate add-on hydroxychloroquine suggested that this combination could be a superior option for these patients.

There were several limitations with this study. First, unlike in a randomized-controlled trial, selection bias was inevitable in this real-world analysis. Although adjusted by propensity score matching with the baseline disease activity, GCs, and previous DMARDs exposure, the possibility of other potential confounders that exerted impact on the outcome measurements could not be excluded. What's more, patients did not strictly follow the visit schedules in the study. Consequently, only baseline, 12-month, and 24-month data were included for the analysis of the secondary outcomes and we allowed a 2-month window. Second, as a single center cohort study in China, caution should be taken when extrapolating our results to a larger population or area.

\section{Conclusion}

In conclusion, methotrexate plus hydroxychloroquine was seemingly better in terms of remission response with a favorable cost-effectiveness for established RA patients with inadequate response to MTX monotherapy in a Chinese cohort, compared with the combination of methotrexate and leflunomide.

\section{Data Availability Statement}

The datasets generated and/or analyzed during the current study are available from the corresponding author on reasonable request.

\section{Ethics Approval and Consent to Participate}

The study was established following the OPCSP trial (ClinicalTrials.gov ID: NCT03024307) and performed in compliance with the Declaration of Helsinki. The research 
protocol was approved by Shanghai Jiaotong University School of Medicine, Renji Hospital Ethics Committee (approval No.[2016]216K), in accordance with the Declaration of Helsinki. All participating patients provided written informed consent.

\section{Acknowledgments}

The authors thank all the rheumatologists, nurses, and patients who participated in this study.

\section{Funding}

This study was funded by National Natural Science Foundation Youth Project (71804109), Cooperative Research Project of Innovation of Translational Medicine from Shanghai Jiaotong University (TM201807), 2018 Pujiang Outstanding Youth Project of Renji Hospital South Campus (RJPJYQ2018), and Minhang District Municipal Commission of Health scientific research project (2019MW33). Le Zhang, Fangfang Chen, and Shikai Geng are co-first authors for this study.

\section{Disclosure}

The authors declare that they have no conflict of interests.

\section{References}

1. Smolen JS, Landewe RBM, Bijlsma JWJ, et al. EULAR recommendations for the management of rheumatoid arthritis with synthetic and biological disease-modifying antirheumatic drugs: 2019 update. Ann Rheum Dis. 2020;79(6):685-699. doi:10.1136/annrheumdis-2019-216655

2. Rohr MK, Mikuls TR, Cohen SB, Thorne JC, O'Dell JR. Underuse of methotrexate in the treatment of rheumatoid arthritis: a national analysis of prescribing practices in the US. Arthritis Care Res (Hoboken). 2017;69(6):794-800. doi:10.1002/acr.23152

3. van Vollenhoven RF, Ernestam S, Geborek P, et al. Addition of infliximab compared with addition of sulfasalazine and hydroxychloroquine to methotrexate in patients with early rheumatoid arthritis (Swefot trial): 1-year results of a randomised trial. Lancet. 2009;374 (9688):459-466. doi:10.1016/s0140-6736(09)60944-2

4. Moreland LW, O'Dell JR, Paulus HE, et al. A randomized comparative effectiveness study of oral triple therapy versus etanercept plus methotrexate in early aggressive rheumatoid arthritis: the treatment of early aggressive rheumatoid arthritis trial. Arthritis Rheum. 2012;64 (9):2824-2835. doi:10.1002/art.34498

5. Smolen JS, Breedveld FC, Burmester GR, et al. Treating rheumatoid arthritis to target: 2014 update of the recommendations of an international task force. Ann Rheum Dis. 2016;75(1):3-15. doi:10.1136/ annrheumdis-2015-207524

6. Ramiro S, Landewe RB, van der Heijde $\mathrm{D}$, et al. Is treat-to-target really working in rheumatoid arthritis? A longitudinal analysis of a cohort of patients treated in daily practice (RA BIODAM). Ann Rheum Dis. 2020;79(4):453-459. doi:10.1136/annrheumdis-2019-216819

7. Chatzidionysiou K, Emamikia S, Nam J, et al. Efficacy of glucocorticoids, conventional and targeted synthetic disease-modifying antirheumatic drugs: a systematic literature review informing the 2016 update of the EULAR recommendations for the management of rheumatoid arthritis. Ann Rheum Dis. 2017;76(6):1102-1107. doi:10.1136/annrheumdis-2016-210711
8. van Vollenhoven RF, Geborek P, Forslind K, et al. Conventional combination treatment versus biological treatment in methotrexate-refractory early rheumatoid arthritis: 2 year follow-up of the randomised, non-blinded, parallel-group Swefot trial. Lancet. 2012;379 (9827):1712-1720. doi:10.1016/s0140-6736(12)60027-0

9. Verschueren P, De Cock D, Corluy L, et al. Effectiveness of methotrexate with step-down glucocorticoid remission induction (COBRA Slim) versus other intensive treatment strategies for early rheumatoid arthritis in a treat-to-target approach: 1-year results of CareRA, a randomised pragmatic open-label superiority trial. Ann Rheum Dis. 2017;76(3):511-520. doi:10.1136/annrheumdis-2016-209212

10. Burmester GR, Kremer JM, Van den Bosch F, et al. Safety and efficacy of upadacitinib in patients with rheumatoid arthritis and inadequate response to conventional synthetic disease-modifying anti-rheumatic drugs (SELECT-NEXT): a randomised, double-blind, placebo-controlled Phase 3 trial. Lancet. 2018;391(10139):2503-2512. doi:10.1016/s01406736(18)31115-2

11. van Vollenhoven RF, Fleischmann R, Cohen S, et al. Tofacitinib or adalimumab versus placebo in rheumatoid arthritis. $N$ Engl $J$ Med. 2012;367(6):508-519. doi:10.1056/NEJMoa1112072

12. Porter D, van Melckebeke J, Dale J, et al. Tumour necrosis factor inhibition versus rituximab for patients with rheumatoid arthritis who require biological treatment (ORBIT): an open-label, randomised controlled, non-inferiority, trial. Lancet. 2016;388(10041):239-247. doi:10.1016/s0140-6736(16)00380-9

13. Burmester GR, Lin Y, Patel R, et al. Efficacy and safety of sarilumab monotherapy versus adalimumab monotherapy for the treatment of patients with active rheumatoid arthritis (MONARCH): a randomised, double-blind, parallel-group Phase III trial. Ann Rheum Dis. 2017;76 (5):840-847. doi:10.1136/annrheumdis-2016-210310

14. Zhang L, Lu GH, Ye S, Wu B, Shen Y, Li T. Treatment adherence and disease burden of individuals with rheumatic diseases admitted as outpatients to a large rheumatology center in Shanghai, China. Patient Prefer Adherence. 2017;11:1591-1601. doi:10.2147/PPA.S144624

15. Hay SI, Abajobir AA, Abate KH, et al. Global, regional, and national disability-adjusted life-years (DALYs) for 333 diseases and injuries and healthy life expectancy (HALE) for 195 countries and territories, 1990-2016: a systematic analysis for the global burden of disease study 2016. Lancet. 2017;390(10100):1260-1344. doi:10.1016/ s0140-6736(17)32130-x

16. Bansback N, Phibbs CS, Sun H, et al. Triple therapy versus biologic therapy for active rheumatoid arthritis: a cost-effectiveness analysis. Ann Intern Med. 2017;167(1):8-16. doi:10.7326/M16-0713

17. O'Dell JR, Mikuls TR, Taylor TH, et al. Therapies for active rheumatoid arthritis after methotrexate failure. $N$ Engl J Med. 2013;369 (4):307-318. doi:10.1056/NEJMoa1303006

18. Bharat S, Srivastava B, Singh P, Bhardwaj R, Gaur S. Efficacy of combination therapy of methotrexate with hydroxychloroquine or sulfasalazine in rheumatoid arthritis patients in kumaon region: a comparative study. J Evol Med Dent Sci. 2016;5(51):3303-3307. doi:10.14260/jemds/2016/765

19. O’Dell JR, Leff R, Paulsen G, et al. Treatment of rheumatoid arthritis with methotrexate and hydroxychloroquine, methotrexate and sulfasalazine, or a combination of the three medications: results of a two-year, randomized, double-blind, placebo-controlled trial. Arthritis Rheum. 2002;46(5):1164-1170. doi:10.1002/art.10228

20. Aletaha D, Stamm T, Kapral T, et al. Survival and effectiveness of leflunomide compared with methotrexate and sulfasalazine in rheumatoid arthritis: a matched observational study. Ann Rheum Dis. 2003;62(10):944-951. doi:10.1136/ard.62.10.944

21. Jaimes-Hernández J, Meléndez-Mercado CI, Mendoza-Fuentes A, Aranda-Pereira P, Castañeda-Hernández G. Efficacy of leflunomide $100 \mathrm{mg}$ weekly compared to low dose methotrexate in patients with active rheumatoid arthritis. double blind, randomized clinical trial. Reumatol Clin. 2012;8(5):243-249. doi:10.1016/j.reumae.2012.07. 005 
22. Strand V, Cohen S, Schiff M, et al. Treatment of active rheumatoid arthritis with leflunomide compared with placebo and methotrexate. Leflunomide rheumatoid arthritis investigators group. Arch Intern Med. 1999;159(21):2542-2550. doi:10.1001/archinte.159.21.2542

23. Ebina K, Hashimoto M, Yamamoto W, et al. Drug tolerability and reasons for discontinuation of seven biologics in 4466 treatment courses of rheumatoid arthritis-the ANSWER cohort study. Arthritis Res Ther. 2019;21(1):91. doi:10.1186/s13075-019-1880-4

24. Grijalva CG, Chung CP, Arbogast PG, Stein CM, Mitchel EF Jr., Griffin MR. Assessment of adherence to and persistence on disease-modifying antirheumatic drugs (DMARDs) in patients with rheumatoid arthritis. Med Care. 2007;45(10Supl 2):S66-76. doi:10.1097/MLR.0b013e318041384c

25. Aletaha D, Neogi T, Silman AJ, et al. 2010 rheumatoid arthritis classification criteria: an American college of rheumatology/european league against rheumatism collaborative initiative. Ann Rheum Dis. 2010;69(9):1580-1588. doi:10.1136/ard.2010.138461

26. Liu GG, Wu H, Li M, Gao C, Luo N. Chinese time trade-off values for EQ-5D health states. Value Health. 2014;17(5):597-604. doi:10.1016/j.jval.2014.05.007

27. Bergstra SA, Winchow LL, Murphy E, et al. How to treat patients with rheumatoid arthritis when methotrexate has failed? The use of a multiple propensity score to adjust for confounding by indication in observational studies. Ann Rheum Dis. 2019;78(1):25-30. doi:10.1136/annrheumdis-2018-213731

28. Vastesaeger N, Xu S, Aletaha D, St Clair EW, Smolen JS. A pilot risk model for the prediction of rapid radiographic progression in rheumatoid arthritis. Rheumatology (Oxford). 2009;48(9):1114-1121. doi:10.1093/rheumatology/kep155

29. Visser K, Goekoop-Ruiterman YP, de Vries-bouwstra JK, et al. A matrix risk model for the prediction of rapid radiographic progression in patients with rheumatoid arthritis receiving different dynamic treatment strategies: post hoc analyses from the BeSt study. Ann Rheum Dis. 2010;69(7):1333-1337. doi:10.1136/ard.2009.121160

30. Lamb SE, Williamson EM, Heine PJ, et al. Exercises to improve function of the rheumatoid hand (SARAH): a randomised controlled trial. Lancet. 2015;385(9966):421-429. doi:10.1016/s0140-6736(14) 60998-3

31. Klareskog L, van der Heijde D, de Jager JP, et al. Therapeutic effect of the combination of etanercept and methotrexate compared with each treatment alone in patients with rheumatoid arthritis: double-blind randomised controlled trial. Lancet. 2004;363 (9410):675-681. doi:10.1016/s0140-6736(04)15640-7

32. Emery P, Breedveld FC, Hall S, et al. Comparison of methotrexate monotherapy with a combination of methotrexate and etanercept in active, early, moderate to severe rheumatoid arthritis (COMET): a randomised, double-blind, parallel treatment trial. Lancet. 2008;372(9636):375-382. doi:10.1016/s0140-6736(08)61000-4
33. van der Heijde D, Klareskog L, Rodriguez-Valverde V, et al. Comparison of etanercept and methotrexate, alone and combined, in the treatment of rheumatoid arthritis: two-year clinical and radiographic results from the TEMPO study, a double-blind, randomized trial. Arthritis Rheum. 2006;54(4):1063-1074. doi:10.1002/art.21655

34. Kay J, Matteson EL, Dasgupta B, et al. Golimumab in patients with active rheumatoid arthritis despite treatment with methotrexate: a randomized, double-blind, placebo-controlled, dose-ranging study. Arthritis Rheum. 2008;58(4):964-975. doi:10.1002/art.23383

35. Khraishi M, Ivanovic J, Zhang Y, et al. Long-term etanercept retention patterns and factors associated with treatment discontinuation: a retrospective cohort study using Canadian claims-level data. Clin Rheumatol. 2018;37(9):2351-2360. doi:10.1007/s10067-018-4141-z

36. Kerschbaumer A, Sepriano A, Smolen JS, et al. Efficacy of pharmacological treatment in rheumatoid arthritis: a systematic literature research informing the 2019 update of the EULAR recommendations for management of rheumatoid arthritis. Ann Rheum Dis. 2020;79 (6):744-759. doi:10.1136/annrheumdis-2019-216656

37. Kameda H, Fujii T, Nakajima A, et al. Japan college of rheumatology guideline for the use of methotrexate in patients with rheumatoid arthritis. Mod Rheumatol. 2019;29(1):31-40. doi:10.1080/14397595. 2018.1472358

38. Carmichael SJ, Beal J, Day RO, Tett SE. Combination therapy with methotrexate and hydroxychloroquine for rheumatoid arthritis increases exposure to methotrexate. J Rheumatol. 2002;29(10):2077-2083.

39. Jalal H, O’Dell JR, Bridges SL Jr., et al. Cost-effectiveness of triple therapy versus etanercept plus methotrexate in early aggressive rheumatoid arthritis. Arthritis Care Res (Hoboken). 2016;68 (12):1751-1757. doi:10.1002/acr.22895

40. van der Velde G, Pham B, Machado M, et al. Cost-effectiveness of biologic response modifiers compared to disease-modifying antirheumatic drugs for rheumatoid arthritis: a systematic review. Arthritis Care Res (Hoboken). 2011;63(1):65-78. doi:10.1002/acr.20338

41. Ghabri S, Lam L, Bocquet F, Spath HM. Systematic literature review of economic evaluations of biological treatment sequences for patients with moderate to severe rheumatoid arthritis previously treated with disease-modifying anti-rheumatic drugs. Pharmacoeconomics. 2020;38(5):459-471. doi:10.1007/s40273-020-00887-6

42. Hsieh PH, Wu O, Geue C, McIntosh E, McInnes IB, Siebert S. Economic burden of rheumatoid arthritis: a systematic review of literature in biologic era. Ann Rheum Dis. 2020;79(6):771-777. doi:10.1136/annrheumdis-2019-216243

43. Pazmino S, Boonen A, Stouten V, et al. Two-year cost-effectiveness of different COBRA-like intensive remission induction schemes in early rheumatoid arthritis: a piggyback study on the pragmatic randomised controlled CareRA trial. Ann Rheum Dis. 2020;79 (5):556-565. doi:10.1136/annrheumdis-2019-216874
Journal of Inflammation Research

\section{Publish your work in this journal}

The Journal of Inflammation Research is an international, peerreviewed open-access journal that welcomes laboratory and clinical findings on the molecular basis, cell biology and pharmacology of inflammation including original research, reviews, symposium reports, hypothesis formation and commentaries on: acute/chronic inflammation; mediators of inflammation; cellular processes; molecular mechanisms; pharmacology and novel anti-inflammatory drugs; clinical conditions involving inflammation. The manuscript management system is completely online and includes a very quick and fair peerreview system. Visit http://www.dovepress.com/testimonials.php to read real quotes from published authors. 